\title{
Stereotactic Body Radiotherapy for Low- Risk Prostate Cancer: A Ten-Year Analysis
}

\author{
Alan Katz ${ }^{1}$ \\ 1. Flushing radiation \\ $\square$ Corresponding author: Alan Katz, akatzmd@msn.com \\ Disclosures can be found in Additional Information at the end of the article
}

\section{Abstract \\ Objective}

This study represents the first 10 year analysis of the efficacy and toxicity of stereotactic body radiotherapy (SBRT) in the treatment of early low-risk prostate cancer.

\section{Materials and methods}

Two hundred and thirty males were treated with Cyberknife SBRT to a dose of 35 Gray (Gy) or $36.25 \mathrm{~Gy}$ in five consecutive days. All patients had a Gleason score of six and a PSA < 10ng/ml. Median follow-up is nine years. The median age was 69.5 years and median prostate specific antigen (PSA) was $5.6 \mathrm{ng} / \mathrm{ml}$. The treatment was delivered with homogeneous planning with a dose prescription of $82-87 \%$ of the maximum dose to cover the planning target volume (PTV).

\section{Results}

Ten-year biochemical disease free survival was 93\% with either dose. Local control was $98.4 \%$. Median prostate specific antigen (PSA) dropped to 0.1 by five years and has remained there. Toxicity was mild with $10 \%$ of patients having Grade two-three late urinary toxicity and $4 \%$ having the late grade two rectal toxicity. Mean Expanded Prostate Cancer Index Composite (EPIC) Quality of Life (QOL) scores declined initially for bowel and urinary domains, but recovered to baseline, where they remain. EPIC sexual scores have declined by $40 \%$.

\section{Discussion/Conclusions}

Stereotactic body radiotherapy to a dose of $35 \mathrm{~Gy}-36.25 \mathrm{~Gy}$ is an effective treatment for early low-risk prostate cancer, with acceptably low toxicity. There appears to be no benefit to increasing the dose beyond $35 \mathrm{~Gy}$. Ten-year biochemical disease free survival appears to be higher than with standard intensity modulated radiotherapy (IMRT).

Received 07/31/2017

Review began 09/04/2017 Review ended 09/05/2017 Published 09/09/2017

(C) Copyright 2017

Katz. This is an open access article distributed under the terms of the Creative Commons Attribution License CC-BY 3.0., which permits unrestricted use, distribution, and reproduction in any medium, provided the original author and source are credited.
Categories: Radiation Oncology, Urology

Keywords: stereotactic radiotherapy, prostate, carcinoma

\section{Introduction}

Over the last 10 years, many reports of stereotactic body radiotherapy (SBRT) treatments for early prostate cancer, in which high fractional doses are delivered in four or five fractions, have appeared in the literature [1-7]. The effects of doses of $35 \mathrm{~Gy}$ to $50 \mathrm{~Gy}$ in five fractions have been assessed. With follow-up up to seven years, these studies, mostly involving low- and intermediate- risk patients have shown excellent biochemical control with low rates of toxicity. 
The sensitivity of the prostate cancer to dose per fraction probably accounts for these favorable results [8]. Although the outcomes to date have been favorable, a legitimate concern is that the reported follow-up is not long enough to identify all the benefits and pitfalls of this treatment. In this study, we address these concerns by reporting a large cohort of low-risk patients with up to 10-years of follow-up.

\section{Materials And Methods}

From 2006-2008, we treated 230 low-risk prostate cancer patients with CyberKnife SBRT. The patients received a dose of 3500 to $3625 \mathrm{cGy}$ in five daily fractions. All patients had a Gleason score of six. The median prostate specific antigen (PSA) was $5.6 \mathrm{ng} / \mathrm{ml}$. The median age was 69.5 years (range $47-86$ years). Ninety percent of the patients were T1c and 10\% were T2a. The patients had one to nine positive cores on 12-core biopsy. The median prostate volume was 45 cc (range 18-103 cc). All patients were planned with a computerized tomography (CAT) scan fused with a magnetic resonance imaging (MRI) scan. The first 42 patients received a dose of 35 Gy in five daily fractions to the PTV and the rest received $36.25 \mathrm{~Gy}$, also in five daily fractions. The treatment dose was defined to cover $95 \%$ of the PTV. The PTV was defined as a 5-m expansion of the actual prostate volume, reduced to $3 \mathrm{~mm}$ toward the rectum. Seminal vesicles were included in the treatment volume for lesions at the base. The dose prescribed was $82-87 \%$ of the maximum dose in the prostate. The mean dextrose (D50) to the rectum and bladder was $43 \%$ of the maximum dose. All patients received $500 \mathrm{mg}$ of Amifostine solution instilled into the rectum 15 minutes before the treatment.

The median follow-up for the entire cohort was 108 months (zero-120 months). In general, PSA values were obtained at baseline, and prospectively at three-month post-treatment intervals during the first two years and at six-months intervals thereafter. The PSA relapse definition used was the current adopted standard of care Phoenix definition (i.e., nadir + 2) [9]. Biochemical disease-free survival (BDFS) was calculated with the Kaplan-Meier method. A benign PSA bounce was recorded when PSA rose by $>0.2 \mathrm{ng} / \mathrm{mL}$ above the post-treatment nadir and subsequently returned to nadir levels or below.

\section{Toxicity}

Acute and late genitourinary (GU) and gastrointestinal (GI) toxicity were scored according to the criteria set by the Radiation Therapy Oncology Group (RTOG) [10].

\section{Quality of life}

Prior to the treatment, all the patients completed the initial Expanded Prostate Cancer Index Composite (EPIC) questionnaire to evaluate urinary, bowel, and sexual quality of life (QOL) [11]. At each subsequent time, the patients were requested to fill out the EPIC questionnaire to assess follow-up QOL.

\section{Statistical analysis}

The primary endpoint of the study was interval to BDFS. Kaplan-Meier survival method was used to estimate BDFS and log-rank p-values were used to compare the distributions. One tailed test was used to determine $\mathrm{p}$ value for the difference in toxicity.

\section{Results}

The 10-year actuarial biochemical disease free survival was 93.7\% (Figure 1). Local control was 98.4\%. The median PSA level dropped to $0.1 \mathrm{ng} / \mathrm{ml}$ at 48 months. At 10 -years, the median PSA remains at $0.1 \mathrm{ng} / \mathrm{ml}$ (Figure 2). There was no difference in control or median PSA between the two doses of $35 \mathrm{~Gy}$ and $36.25 \mathrm{~Gy}$. Twenty-one percent of the patients experienced a PSA 


\section{Biochemical Disease Free Survival}

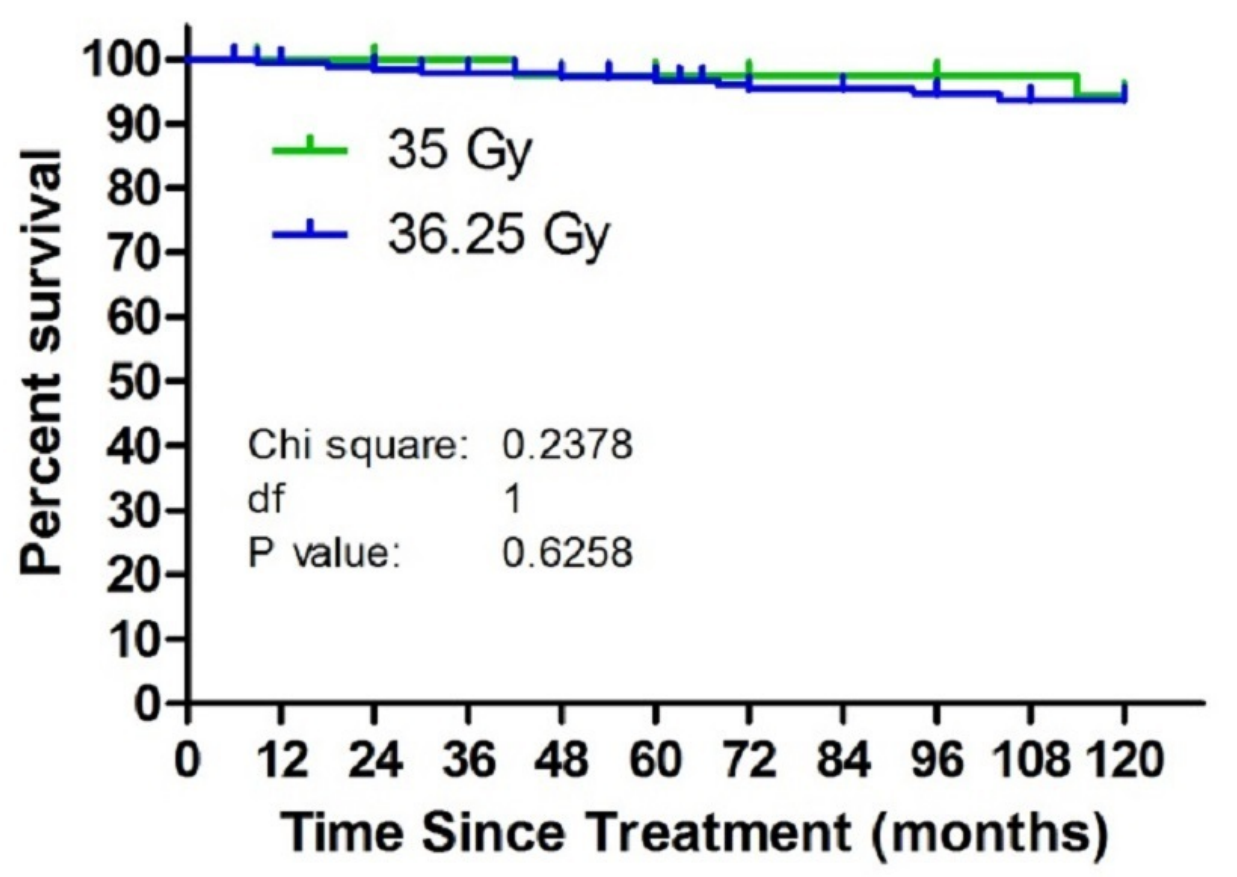

Patients at Risk
\begin{tabular}{|c|ccccccccccc|}
\hline Months & 0 & 12 & 24 & 36 & 48 & 60 & 72 & 84 & 96 & 108 & 120 \\
\hline 3500 & 41 & 41 & 40 & 40 & 38 & 37 & 36 & 36 & 35 & 35 & 31 \\
\hline 3625 & 190 & 186 & 182 & 176 & 173 & 161 & 149 & 142 & 132 & 87 & 26 \\
\hline
\end{tabular}

FIGURE 1: Biochemical disease-free survival (BDFS) in the patients treated with 35 or 36.25 Gy. The 10-year Kaplan-Meier estimate of BDFS was $94.4 \%$ for the patients treated with 35 Gy and $93.4 \%$ for the patients treated with $36.25 \mathrm{~Gy}$. 


\section{Cureus}

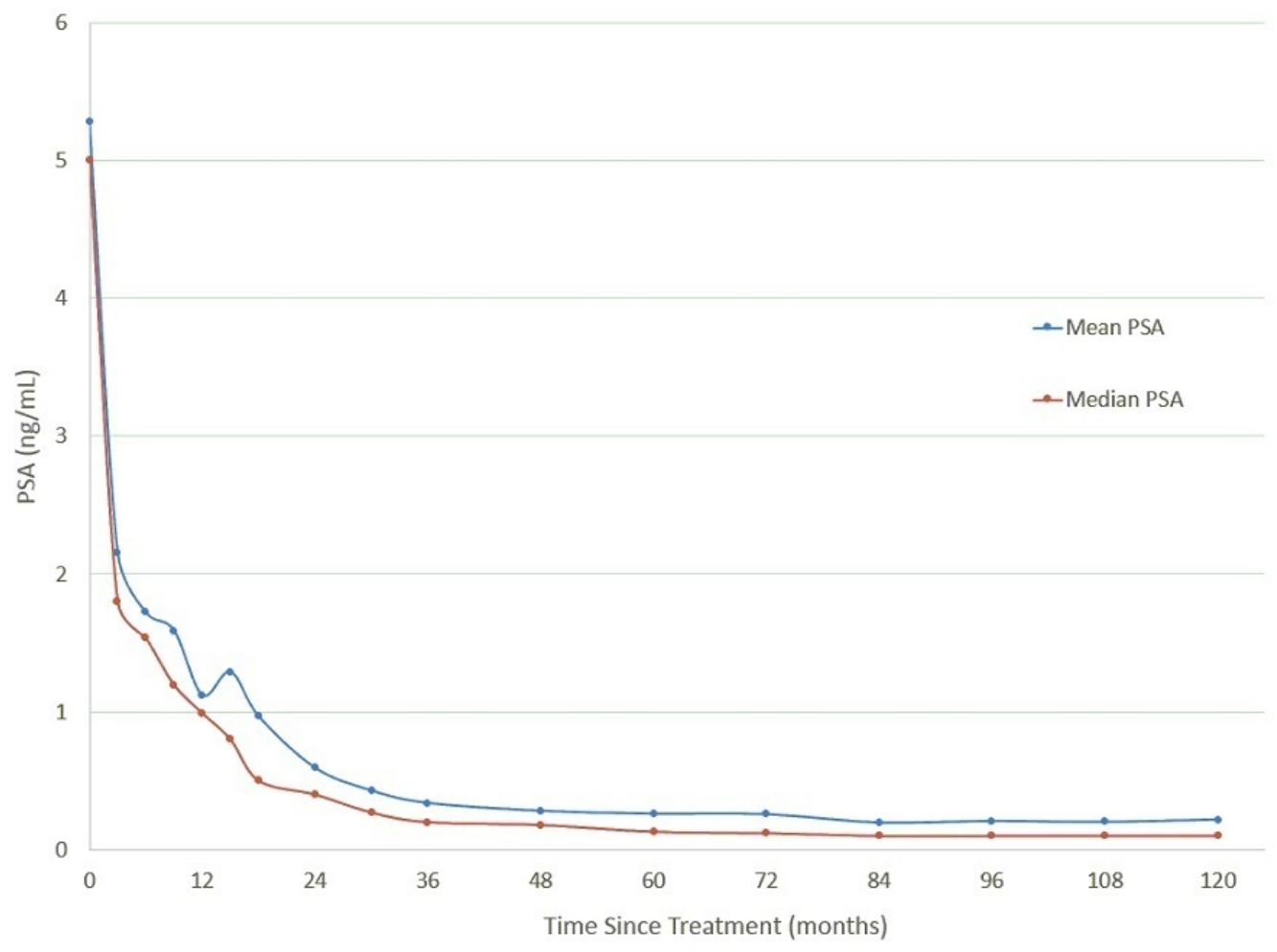

FIGURE 2: Mean (blue) and median (red) prostate specific antigen (PSA) value as a function for months since treatment.

The toxicity was mild. Grade 1-2 acute urinary toxicity was noted in $78 \%$ of the patients; $59 \%$ had acute Grade 1-2 rectal toxicity. There was no Grade 3-4 toxicity. Late urinary toxicity occurred in 9\% (Grade 2) and 3\% (Grade 3) of the patients. Late urinary toxicity Grade 2-3 appeared higher in the high-dose group (4\% at $35 \mathrm{~Gy}$ vs $15 \%$ at $36.25 \mathrm{~Gy}$ ), and this difference approached significance $(\mathrm{p}=0.07)$. Four percent of the patients had Grade 2 rectal toxicity with no Grade 3-4 events. There was no clear difference in late rectal toxicity rates between the two doses. For patients who were potent prior to SBRT, 56\% remained potent at last follow-up.

For urinary (Figure 3) and bowel (Figure 4) EPIC domains, the mean EPIC scores decreased acutely and then gradually rose back to baseline by one-year. After one-year, mean EPIC scores extending, out to eight years did not differ significantly from baseline. The EPIC sexual QOL declined by $23 \%$ at six-12 months and decreased by $38 \%$ by eight years (Figure 5 ). There was no significant difference in EPIC bowel, sexual, or urinary scores between 35 Gy or 36.25 Gy at any point. 


\section{Cureus}

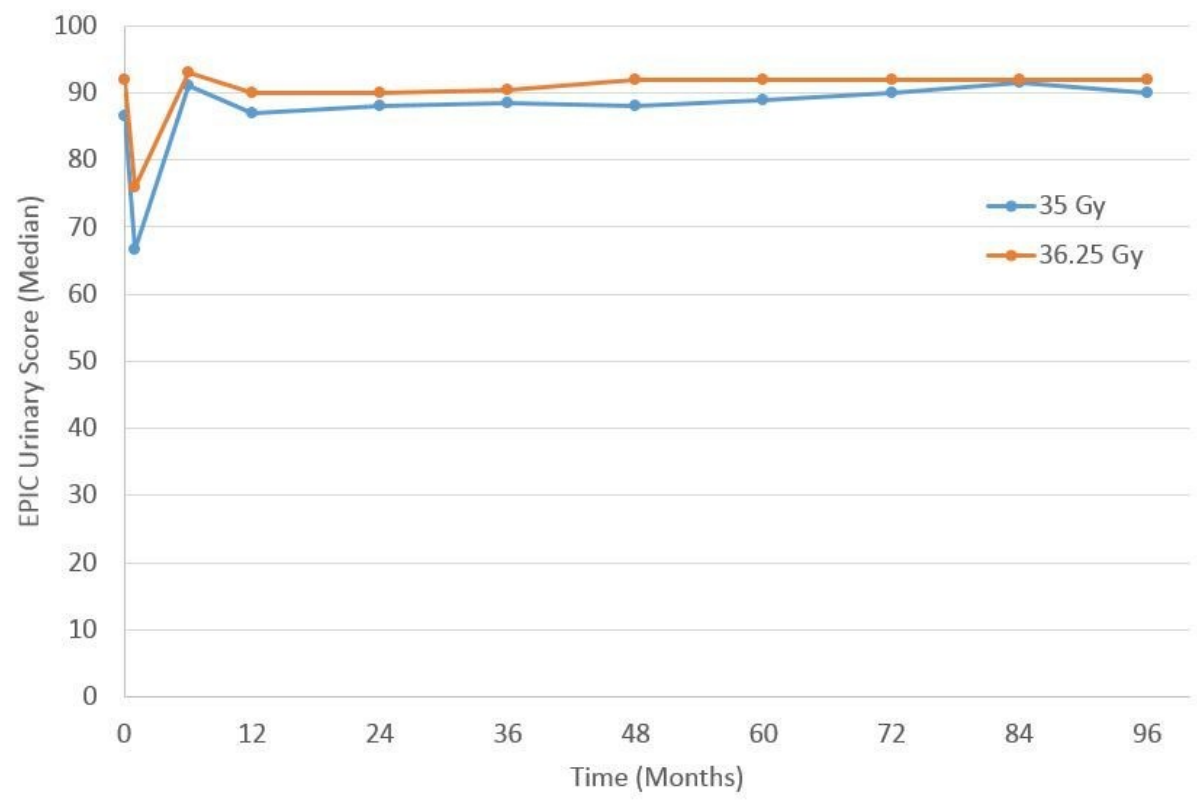

FIGURE 3: Expanded Prostate Cancer Index Composite (EPIC) urinary quality of life as a function of time since the treatment for the 35 and 36.25 Gy cohorts.

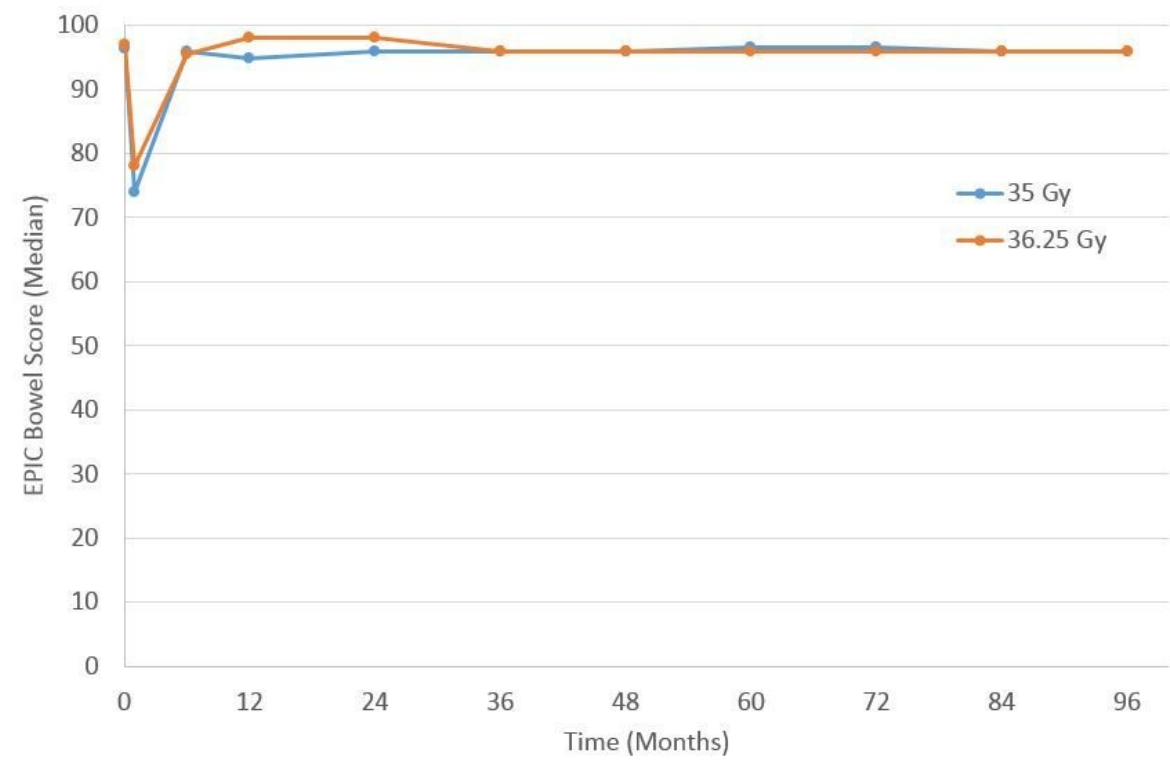

FIGURE 4: Expanded Prostate Cancer Index Composite (EPIC) bowel quality of life as a function of time since the treatment of 35 and 36.25 Gy cohorts. 


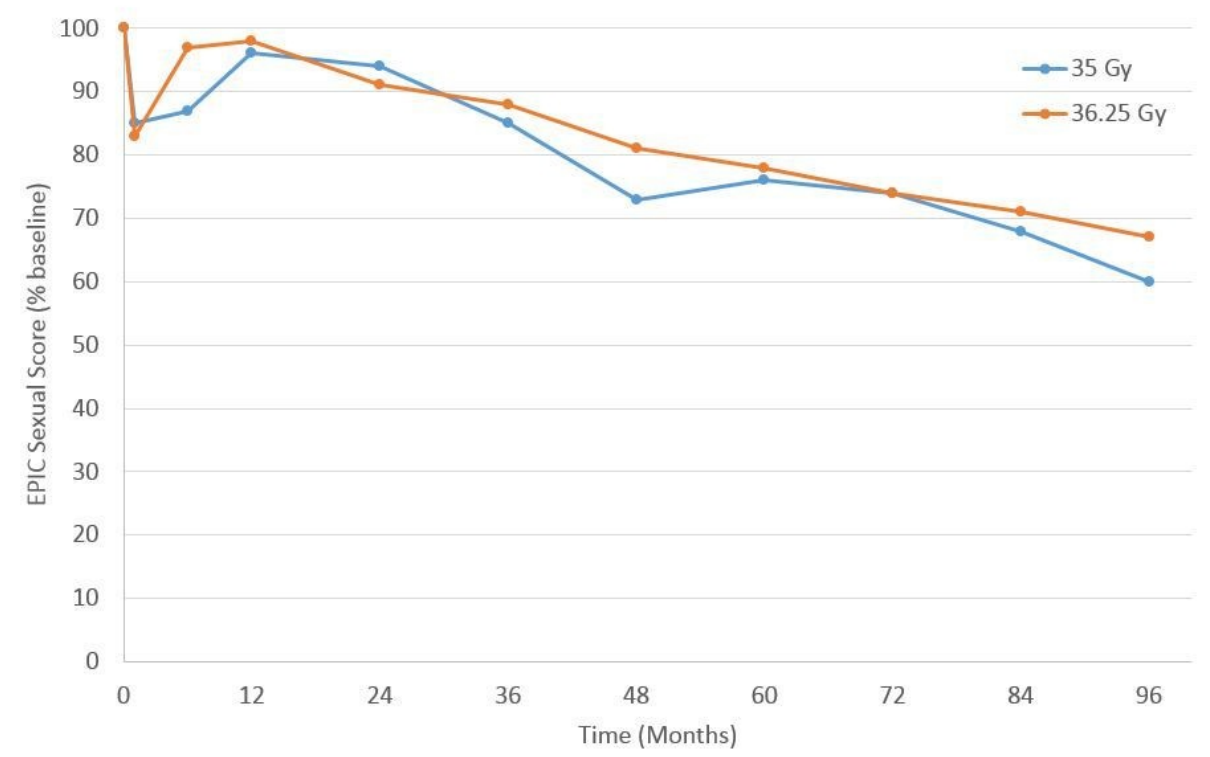

FIGURE 5: Expanded Prostate Cancer Index Composite (EPIC) sexual quality of life for the patients with good pre-treatment function, as a function of time since the treatment of $\mathbf{3 5}$ and 36.25 Gy cohorts.

\section{Discussion}

At ten-years, this report demonstrates excellent long-term control of low-risk prostate cancer with low toxicity. These outcomes compare quite favorably to the patients treated with IMRT to $81 \mathrm{~Gy}$. The two reports of standard IMRT with this long-term follow-up show biochemical control rates of $81-85 \%$ of low-risk patients [12-13]. A ten-year study of such patients with high dose rate (HDR) brachytherapy demonstrated a $92 \%$ control rate, quite similar to our patients [14]. This supports the notion that a dose of $81 \mathrm{~Gy}$ in 45 fractions, or 178 biologically effective dose (BED), may not be enough to maximize tumor control. Since HDR and SBRT seem to yield higher control rates, it is highly likely that the equivalent dose delivered with these two modalities is significantly higher than $81 \mathrm{~Gy}$. This is probably a function of the low alpha-beta ratio of prostate cancer. In fact, an alpha-beta ratio of 1.5 Gy would mean that $35-36.25 \mathrm{~Gy}$ in five fractions yields an equivalent dose of 90-95 Gy at 1.8 Gy per fraction, or 200-212 Gy BED.

If a dose escalation with SBRT delivers higher control rates, the next question is at what dose will the maximum benefit occur. Based on our data, $35 \mathrm{~Gy}$ in five fractions achieves the maximum benefit as our higher dose showed no further benefit. Using an alpha-beta ratio of 1.5 Gy, our 35-Gy dose has a BED of $200 \mathrm{~Gy}$. This is consistent with a recent meta-analysis of prostate cancer patients treated with various radiation modalities, which showed a maximum benefit for $200 \mathrm{BED}$, with only more toxicity for higher doses [15].

Consistent with the above, our data also show higher urinary toxicity with $36.25 \mathrm{~Gy}$, which has a BED of 212 Gy. This strongly suggests that 35 Gy may be the optimal dose to employ in lower risk patients and that higher doses being employed today will only yield more toxicity with no further benefit. Whether higher doses may benefit higher risk patients remains an open question. These results will need to be confirmed by the maturation of other trials and possibly randomized trials between SBRT and other radiation modalities. 


\section{Conclusions}

Stereotactic body radiotherapy (SBRT) of 35-36.25 Gy in five fractions yields excellent control with low toxicity in low-risk prostate cancer patients. Further randomized trials are necessary to compare its efficacy with other forms of radio therapy (RT) and to determine the optimal SBRT dose. This study suggests that SBRT is superior to standard IMRT in terms of control with no increase in late toxicity.

\section{Additional Information \\ Disclosures}

Human subjects: Consent was obtained by all participants in this study. Animal subjects: All authors have confirmed that this study did not involve animal subjects or tissue. Conflicts of interest: In compliance with the ICMJE uniform disclosure form, all authors declare the following: Payment/services info: All authors have declared that no financial support was received from any organization for the submitted work. Financial relationships: All authors have declared that they have no financial relationships at present or within the previous three years with any organizations that might have an interest in the submitted work. Other relationships: All authors have declared that there are no other relationships or activities that could appear to have influenced the submitted work.

\section{References}

1. Chen LN, Suy S, Uhm S, et al.: Stereotactic body radiation therapy (SBRT) for clinically localized prostate cancer: the Georgetown University experience. Radiat Oncol. 2013, 8:58. 10.1186/1748-717x-8-58

2. Freeman DE, King CR: Stereotactic body radiotherapy for low-risk prostate cancer: Five-year outcomes. Radiat Oncol. 2011, 6:3. doi:10.1186/1748-717X-6-3

3. Katz AJ, Kang J: Stereotactic body radiotherapy as treatment for organ confined low- and intermediate-risk prostate carcinoma, a 7-year study. Front Oncol. 2014, 4:240. 10.3389/fonc. 2014.00240

4. Katz AJ, Santoro M, Diblasio F, et al.: Stereotactic body radiotherapy for localized prostate cancer: disease control and quality of life at 6 years. Radiat Oncol. 2013, 8:118. 10.1186/1748$717 \mathrm{x}-8-118$

5. King CR, Brooks JD, Gill H, et al.: Long-term outcomes from a prospective trial of stereotactic body radiotherapy for low-risk prostate cancer. Int J Radiat Oncol Biol Phys. 2012, 82:877882. 10.1016/j.ijrobp.2010.11.054

6. King CR, Freeman D, Kaplan I, et al.: Stereotactic body radiotherapy for localized prostate cancer: pooled analysis from a multi-institutional consortium of prospective phase II trials. Radiother Oncol. 2013, 109:217-221. 10.1016/j.radonc.2013.08.030

7. Oliai C, Lanciano R, Sprandio B, et al.: Stereotactic body radiation therapy for the primary treatment of localized prostate cancer. J Radiat Oncol. 2013, 2:63-70. 10.1007/s13566-0120067-2

8. Miralbell R, Roberts SA, Zubizarreta E, et al.: Dose-fractionation sensitivity of prostate cancer deduced from radiotherapy outcomes of 5,969 patients in seven international institutional datasets: alpha/beta = 1.4 (0.9-2.2) Gy. Int J Radiat Oncol Biol Phys. 2012, 82:17-24. 10.1016/j.ijrobp.2010.10.075

9. Roach M, Hanks G, Thames H, et al.: Defining biochemical failure following radiotherapy with or without hormonal therapy in men with clinically localized prostate cancer: recommendations of the RTOG-ASTRO Phoenix Consensus Conference. Int J Radiat Oncol Biol Phys. 2006, 65:965-974. 10.1016/j.ijrobp.2006.04.029

10. Cox JD, Stetz J, Pajak TF: Toxicity criteria of the Radiation Therapy Oncology Group (RTOG) and the European organization for research and treatment of cancer (EORTC). Int J Radiat Oncol Biol Phys. 1995, 31:1341-1346. 10.1016/0360-3016(95)00060-c

11. Wei JT, Dunn RL, Litwin MS, et al.: Development and validation of the expanded prostate cancer index composite (EPIC) for comprehensive assessment of health-related quality of life 


\section{Cureus}

in men with prostate cancer. Urology. 2000, 56:899-905.

12. Alicikus ZA, Yamada Y, Zhang Z, et al.: Ten-year outcomes of high-dose, intensity-modulated radiotherapy for localized prostate cancer. Cancer. 2011, 117:1429-1437. 10.1002/cncr.25467

13. Weller MA, Reddy CA, Kittel J, et al.: Comparison of Outcomes Between Brachytherapy and Intensity Modulated Radiation Therapy in High-Risk Prostate Cancer in American Society for Radiation Oncology 56th Annual Meeting. San Francisco, CA; 2014.

14. Demanes DJ, Rodriguez RR, Schour L, et al.: High-dose-rate intensity-modulated brachytherapy with external beam radiotherapy for prostate cancer: California endocurietherapy's 10-year results. Int J Radiat Oncol Biol Phys. 2005, 61:1306-1316. 10.1016/j.ijrobp.2004.08.014

15. Zaorsky NG, Palmer JD, Hurwitz MD, et al.: What is the ideal radiotherapy dose to treat prostate cancer? A meta-analysis of biologically equivalent dose escalation. Radiother Oncol. 2015, 115:295-300. 10.1016/j.radonc.2015.05.011 